\title{
Aging and Nutrition: A Review Article
}

\author{
Shruti Singh $1 \&$ Sunita Mishra 2 \\ 1( Research Scholar, Department of Human Development \& Family Studies,School for Home Sciences, \\ Babasaheb Bhimrao Ambedkar University, India) \\ 2 (Dean \& HOD, Department of Human Development \& Family Studies, School for Home Sciences, Babasaheb \\ Bhimrao Ambedkar University, India)
}

\begin{abstract}
Aging is defined as a genetic physiological process associated with morphological and functional changes in cellular and extracellular components aggravated by injury trough out life and resulting in a progressive imbalance of the control regulatory systems of the organism, including hormonal, autocrine, neuroendocrine and immune homeostatic mechanisms. Nutrition has solid potential in preserving youth and vitality. Calcium \& Vitamin-D prevents osteoporosis and maintain healthy bones, Vitamin B12 to build red blood cells and maintain healthy nerves, Zinc to help compensate for lowered immunity due to ageing, Potassium is needed especially in the presence of high blood pressure, Folic acid \& Vitamin- B, help in DNA and red blood cells formation, can lower the levels of haemocysteine(associated with heart disease), Fiber prevents constipation, Omega-3 fatty acids prevents cardiac death by blocking fatal heart rhythms and keeps arteries healthy and reduces the sickness of platelets in the blood, vitamin- $C$ and $E \&$ the phytochemicals; lutein, zeaxanthin \& beta-carotene prevent or slow the onset of age-related macular degeneration and Alzheimer's disease, water keeps the body hydrated and cleansed. Avocado, Berries, Cruciferous vegetables, garlic, ginger, nuts, soya, watermelon, salmon, yogurt, turmeric, grapes, broccoli, whole grains, olive oils, tomatoes, fish, green tea, dark chocolates, red wine, pomegranates are some top anti aging foods.
\end{abstract}

Key Words: Aging, Nutrition, Antioxidants, Anti-aging foods

\section{What Is Aging?}

Aging is defined as a genetic physiological process associated with morphological and functional changes in cellular and extracellular components aggravated by injury throughout life and resulting in a progressive imbalance of the control regulatory systems of the organism, including hormonal, autocrine, neuroendocrine and immune homeostatic mechanisms. Ageing is the process of growing older; it includes a reduction in strength, endurance, speed of reaction, agility, basal metabolism, sexual activity and hearing acuity. The bones are more brittle, the skin drier and less elastic and the teeth are shed.

There are five criteria's for aging;

Cumulative: Effects of ageing increase with time.

Universal: All members of a species display signs of ageing. Progressive: Ageing is a series of gradual changes.

Intrinsic: Changes would take place even in a "perfect" environment.

Deleterious: Changes which occur compromise normal biological functions. (Source: Barja G.1994)

\section{The Aging Body}

There are several changes which take place in the aging body and most of them are depleting or deteriorating.

\section{SENSORY CHANGES:}

- Decline in sight and peripheral vision, hearing, smell and taste.

\section{STRUCTURAL CHANGES:}

- As we age, we lose lean body mass. Reduced muscle mass includes skeletal muscle, smooth muscle and muscle that affects vital organ function, with loss of cardiac muscle perhaps the most important. Cardiac capacity can be reduced and cardiac function impaired by chronic diseases such as atherosclerosis, hypertension or diabetes.

- Changes occur in the kidneys, lungs and liver, and in ability to generate new protein tissue. Aging can slow the immune system's response in making antibodies.

- The loss of lean body mass may be the decrease in basal energy metabolism. To avoid gaining weight, we must reduce calorie intake or increase activity. The goal is energy balance.

- Reduced body water - 72 percent of total body water is in lean muscle tissue. 
- Total body fat typically increases with age. Fat tends to concentrate in the trunk and as fat deposits around the vital organs. However, in more advanced years, weight often declines.

- Finally, we lose bone density. After menopause, women tend to lose bone mass at an accelerated rate. There is high incidence of osteoporosis.

Aging is a multi factorial biochemical and physiological process that leads to overall cellular breakdown and death. Aging not only alters our physical appearance due to changes in skin, bones, and muscle tone, but also affects our internal organs. The heart and immune system become less efficient, and diseases that are rare in young people become increasingly more prevalent in older adults. . (Source: Harman D. 1981)

\title{
III. How Does Nutrition Slow Down The Rate Of Aging?
}

Most of us are keen to stay looking as fresh and young as possible, and although there's no way to pause or turn back the clock, there are few age-proof strategies we can employ to make sure we stay looking good. Maintaining a healthy and well-balanced diet is one approach, and the good thing is that there are some foods that are thought to give us extra ammunition when it comes to combating the signs of aging. Special foods contains special nutrients which;

\author{
"Combat muscle fatigue" \\ "Neutralize free radicals" \\ "Reduce DNA damage" \\ "Naturally detoxify" \\ "Add youth, health, and strength"
}

"Super food" is an informal term for foods that benefit the body in many different ways. Super foods help to meet several dietary needs and are great sources of essential vitamins and minerals also fight disease and sickness, making them powerful anti-aging tools. Some top anti aging foods are listed below;

Avocado - It is a good source of healthy monounsaturated fat that may help to reduce level of a bad type of cholesterol in body. Avocado is a good source of vitamin $\mathrm{E}$ and can help to maintain healthy skin and prevent skin aging (vitamin E may also help alleviate menopausal hot flushes). It is rich in potassium which helps prevent fluid retention and high blood pressure.

Berries - All black and blue berries such as blackberries, blueberries, blackcurrants and black grapes contain phytochemicals known as flavonoids and anthocyanins; powerful antioxidants which help to protect the body against damage caused by free radicals and aging, slow the growth of certain cancers and diabetes as well as improve brain function, muscle tone, and balance. Vitamin $\mathrm{C}$ has been shown to reduce the redness of the skin and it helps the skin to recover from sunburns. Strawberries are rich in vitamin C but they do not increase the blood sugar levels as rapidly as some other fruits and berries.

Dark chocolates - Exposure to ultraviolet (UV) radiation ages the skin more quickly. Cocoa beans, from which chocolate is made, have a higher antioxidant capacity than any other food, and the high concentration of antioxidant flavanols in cocoa beans helps reduce inflammation of the skin caused by exposure to UV light. Furthermore, eating dark chocolate can increase circulation in the skin and improve its ability to retain moisture, which can reduce the appearance of wrinkles and help you look younger (source: Williams). But not all chocolate is equal when it comes to anti-aging -- its dark chocolate that provides the greatest benefits. That's because the refining process involved in making other kinds of chocolate actually strips away most of the skinbenefitting antioxidant flavanols.

Cruciferous vegetables - The family of Cruciferous vegetables includes cabbage, cauliflower, broccoli, kale, turnip, sprouts, radish and watercress. Cruciferous vegetables assist the body in its fight against toxins and cancer. Broccoli may protect your skin against the sun damage. It is also rich in skin vitamins such as $\mathrm{C}$, and A. They contain folate, calcium, and other nutrients that support bone health, protect against cognitive decline, and help prevent age-related eye problems. Diets high in cruciferous veggies help reduce risk of memory loss and cancer.

Herbs \& spices - Garlic; A clove of garlic a day (row or cooked) helps to protect the body against cancer and heart disease. The cardio protective effects of garlic are well recorded. One 1994 study in Iowa, USA, of 41,837 women between the age of 55 and 69 suggested that women who ate a clove of garlic at least once a week were 50 percent less likely to develop colon cancer. Another study at Tasgore Medical College in India suggested that garlic reduced cholesterol levels and assisted blood thinning more effectively than aspirin, thus helping to reduce the risk of heart disease (Source: CarmiaBorek. 2006). Turmeric is rich in antioxidants and prevents free radical formation. It also lowers bad cholesterol, neutralizes existing free radicals and may have 
anticancer effects. Ginger, the spicy root can boost the digestive and circulatory systems, which can be useful for older people. Ginger may also help to alleviate rheumatic aches and pains. Cinnamon may help lower blood sugar.

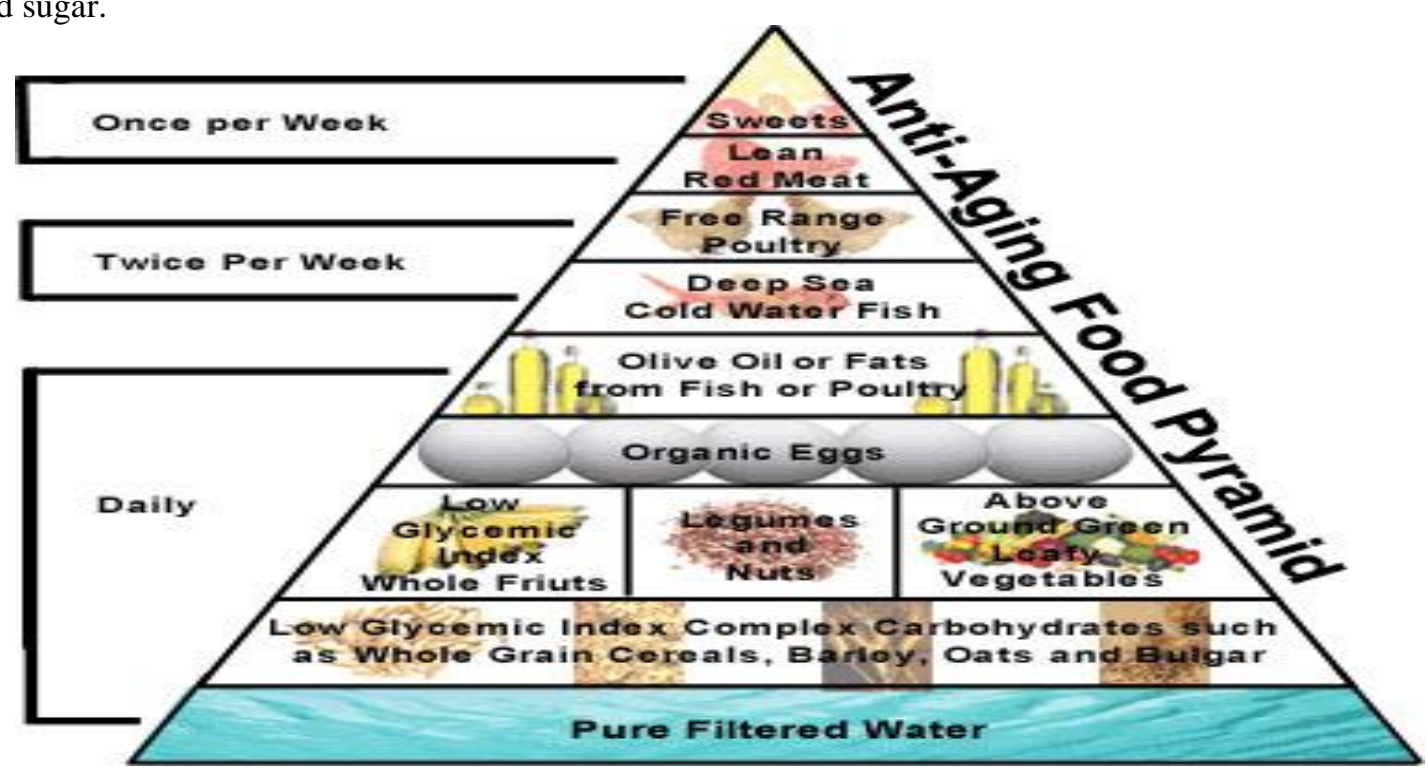

Nuts - Most varieties of nuts are good sources of minerals, particularly walnuts and brazi nuts. Walnuts, although high in calories, are rich in potassium, magnesium, iron, zinc, copper and selenium, can enhance the functioning of digestive and immune systems, improve skin help control prevent cancer. Nuts may also help control cholesterol levels. Never eat rancid nuts, however, as they have been linked to a high incidence of free radicals.

Soya - Menopausal women might find that soya helps to maintain oestrogen levels. Soya may alleviate menopausal hot flush and protect against Alzheimer's disease, osteoporosis and heart disease. Fermented soya products are more easily digested, therefore more nutritional, and do not generally cause food intolerances. You may want to check that soya products have not been genetically modified.

Whole meal pasta and rice - Complex carbohydrates provide a consistent supply of energy throughout the day and should make up the bulk of your diet. Wholemeal pasta is an excellent complex carbohydrate. It is high in fibre and contains twice the amount of iron as normal pasta. Brown rice is another recommended complex carbohydrate, which is high in fibre and B vitamins.

Watermelon - Both the flesh and seeds of the watermelon are nutritious. The flesh contain vitamin A, B and C; the seeds contain selenium, essential fats, zinc and vitamin $\mathrm{E}$, all of which help against free radical damage and aging.

Water - Drink at least 8 glasses of water every day in order to remain healthy. Water helps us to get rid of the toxins and unwanted waste materials from the body.

Fish - Fish provide omega-3 fatty acids that help combat inflammation in the body. People who eat several weekly servings of such fish have a lower risk of Alzheimer's disease and stroke. Omega-3 fatty acids are famous for preventing premature aging. (Source: Marie Cheour. 2010)

Extra Virgin Olive Oil - Consuming too much sugar can cause an inflammatory response in your skin. Extra virgin olive oil is an excellent anti-inflammatory food that should be consumed daily. Olive oil also helps in reducing cholesterol levels, lower rates of cardiovascular disease, cancer, and age-related cognitive decline in people who follow this way of eating.

Oatmeal - Non-instant oatmeal is rich in B vitamins, also increases your blood sugar slowly. This keeps you from getting hungry right after eating. Oatmeal is also an excellent source of magnesium, also vital for healthy skin. 
Yogurt - Yogurt is an excellent source of calcium, vitamin D, and proteins. All of these are important for your skin. In addition, plain yogurt also contains healthy bacteria that make it easier to digest than milk. Yogurts also contain probiotics, which can reduce the inflammation that your skin might have and increase the oxygen levels that your skin receives.

Tomatoes - Certain red fruits, including tomatoes; contain lycopene, an antioxidant compound that helps maintain youthful skin texture and may reduce the risk of some types of cancer (especially prostate, lung, and stomach cancers) and heart disease.

Teas - White and green tea contains the most EGCG, one of the most powerful antioxidants. Numerous studies have linked tea consumption to lower rates of conditions such as heart disease, cancer, and Alzheimer's disease.

Grapes - Grape seeds contain grape seed extract. It is a complex antioxidant; resveratrol that protects collagen from free radicals, and protects blood vessels. Resveratrol has anti-inflammatory and anticoagulant properties, which may explain why red wine and purple grape juice also helps promote heart health.

\section{ANTIOXIDANTS \& HUMAN AGING}

"Theory of aging" given by gerontologists - the so-called "error theory of aging" - aging is primarily caused by external or environmental factors that inflict cellular damage, ultimately leading to organ damage and death. One way these "errors" can occur is through biochemical processes induced by the formation of free radicals, the unstable biochemical entities formed when energy is produced in the cells. While the body can partly neutralize the damaging effects of these radicals, its defences become less efficient with advancing age. This can lead to damaged cells, tissues, and organs, which manifest as the physical declines of aging. Free radicals are thought to cause cellular degeneration by means of a chemical process known as oxidation. Free radical-induced oxidative damage is a major contributor to aging. The levels of endogenous (internally generated) antioxidants - including superoxide dismutase, catalase, and glutathione peroxidise - decline with advancing age. Antioxidants are substances that protect our bodies against free radicals -- unstable molecules that are produced when our bodies break down food or by exposure to pollution and radiation. (Source: Ames BN, Shigenaga MK, 1993)

The imbalance between free radicals and the antioxidants needed to inactivate, or "quench," those leads to a generalized state of oxidative stress that can damage lipids, proteins, DNA, and mitochondria throughout the body. Oxidative stress has been associated with myriad disease processes, including cancer, heart disease, and Alzheimer's. Scientific research suggests that minimizing deleterious free radical reactions by ensuring optimal antioxidant levels may hold the key to extending the healthy human life span. Studies have shown that people who live to be 100 years or older often demonstrate higher blood levels of antioxidants than their much younger counterparts. Furthermore, antioxidants may help protect against mitochondrial dysfunction, another harmful condition that commonly accompanies aging and disease states. (Source: Harman D. 1955)

Numerous antioxidants - lipoic acid, green tea polyphenols, lycopene, and vitamins A, C, and E-are associated with protection against many afflictions that commonly accompany aging, such as Alzheimer's disease, muscle loss (sarcopenia), cataracts, and memory impairment. By protecting against the aberrant biochemical changes that occur with aging, antioxidants may thus represent a veritable fountain of youth. (Source: Zandi PP, Anthony JC, 2004).

Lipoic acid is considered an important antioxidant and crucial for a variety of mitochondrial reactions. Lipoic acid is used to treat liver diseases and polyneuropathies. Recent research has shown that lipoic acid and acetyl-1-carnitine may be a useful adjunct in the fight against pathological and age-related changes seen in the brain. (Source: Barja G. 2004).

Beta-carotene and vitamins $\mathrm{C}$ and $\mathrm{E}$ show great promise in the fight against Alzheimer's disease. Alzheimer's, the most common cause of dementia in adults aged 65 and older, affects more than 15 million people worldwide. One major change that occurs in the brains of Alzheimer's sufferers is generalized oxidative damage to neurons. A study published in the Archives of Neurology in 2004 examined Alzheimer's risk in people who took antioxidant supplements. The study found that "use of vitamin E and vitamin C supplements in combination is associated with reduced prevalence and incidence of alzheimer's disease (Source: Zandi PP, Anthony JC, 2004).

As people age, not only do their bones become brittle, but their muscle tissues shrink and atrophy, a condition known as sarcopenia. When sarcopenia robs people of their ability to walk, climb stairs, or perform the simple task of getting in and out of a chair, it confines them to an unhealthy, sedentary lifestyle. Vitamin E-may help prevent sarcopenia in the elderly. A report from Johns Hopkins examined the relationship between 
plasma levels of antioxidants and muscle strength in women aged 70-79. Higher carotenoid and alpha tocopherol (vitamin E) levels were independently associated with greater muscle strength and concluded that sarcopenia in older adults may result in part from oxidative stress, and that antioxidants may be protective. (Source: Semba RD, Blaum C, 2003)

High intake of carotenoids - the phytocompounds responsible for the red to yellow pigmentation in fruits and vegetables - can protect against various age-related disease states, including vision loss. Two of the most useful carotenoids for combating age-related vision loss caused by cataracts are lutein and zeaxanthin (by protecting the eye lens from the damaging effects of ultraviolet radiation and endogenous free radical formation) (Source: Semba RD, Blaum C, 2003).

Hydrogen sulfide is an antioxidant, it relaxes the arteries and promotes blood flow, and studies have even linked it to cancer defence. The body naturally produces hydrogen sulfide, but adding garlic to your diet causes your cells to produce more of this beneficial compound. Resveratrol, a well recognized antioxidant; may help prevent blood clots, reduce the risk of cancer, decrease inflammation and lower bad cholesterol (Source: Barja G. 2004).

Scientists at the USDA have developed a rating scale that measures the antioxidant content of various plant foods. The scale is called ORAC, which stands for Oxygen Radical Absorbance Capacity. They discovered that a small group of "super foods" have up to twenty times the antioxidant power of other foods. It is recommended that one eat foods containing at least 3,000 ORAC units a day, which is not difficult, since $1 / 2$ cup of blueberries contain 2,400 units.

\section{References}

[1]. Ames BN, Shigenaga MK, Hagen TM. Oxidants, antioxidants, and the degenerative diseases of aging. Proc Natl Acad Sci USA. 1993 Sep 1;90(17):7915-22.

[2]. Barja G. 2004. Free radicals, aging \& antioxidants. Trends Neurosci. 27(10):595-600.

[3]. CarmiaBorek. 2006. "Garlic Reduces Dementia and Heart-Disease Risk," Journal of Nutrition, Vol. 136 (3): $810-812$

[4]. Harman D. 1981. The aging process. Proc Natl Acad Sci USA. 78(11):7124-8.

[5]. Harman, D. 1995. Aging: a theory based on free radical and radiation chemistry. The University of California Radiation Labora tory. Report, No. 3078. Univ. of California, Berkley, CA

[6]. http://www.anti-agingfirewalls.com/2009/09/15/health-and-longevity-benefits-of-dark-chocolate/ cached on September 3, 2012

[7]. http://www.livestrong.com/article/360360-the-top-10-anti-aging-foods-for-the-skin cached on September 12,2012

[8]. http://www.naturalhealthnewsreport.com/ cached on September 11,2012

[9]. http://www.preretirementlife.com/articles/The-Broccoli-Effect-Fight-Aging-and-Help-Your-Heart-290.cfm/ cached on September 3, 2012

[10]. http://www.redorbit.com/news/health/avocado-oil-could-have-anti-aging-disease-fighting-capabilities/ cached on September 11, 2012

[11]. http://www.wellnessresources.com/health/articles/cinnamon_as_an_anti-aging_skin_nutrient/Cached on September 11, 2012

[12]. Marie Cheour. 2010 University of Maryland Medical Center: Omega-3 Fatty Acids. Aging, Vol.4 No.7: 499

[13]. Semba RD, Blaum C, Guralnik JM, et al.2003. Carotenoid and vitamin E status are associated with indicators of sarcopenia among older women living in the community. Aging Clin Exp Res. 15(6):482-7.

[14]. Zandi PP, Anthony JC, Khachaturian AS, et al. 2004. Reduced risk of Alzheimer disease in users of antioxidant vitamin supplements: the Cache County Study. Arch Neurol. 61(1):82-8. 